Original article

\title{
Uncovering interaction between the loops of autonomic regulation of blood circulation from long time series
}

\author{
Viktoriia V. Skazkina ${ }^{1,2}$, Anatoly S. Karavaev ${ }^{1,3}$, Ekaterina I. Borovkova ${ }^{1,2,3}$, Margarita A. Simonyan ${ }^{3}$, \\ Mikhail D. Prokhorov ${ }^{1}$, Vladimir I. Ponomarenko ${ }^{1}$, Elizaveta S. Dubinkina ${ }^{2}$, Boris P. Bezruchko ${ }^{1}$, \\ Vladimir I. Gridnev ${ }^{2,3}$, Anton R. Kiselev ${ }^{2,3}$ \\ ${ }^{1}$ Saratov Branch of the Institute of RadioEngineering and Electronics of Russian Academy of Sciences, Saratov, Russia \\ ${ }^{2}$ Saratov State University, Saratov, Russia \\ ${ }^{3}$ Saratov State Medical University, Saratov, Russia
}

Received 25 June 2020, Revised 14 September 2020, Accepted 2 November 2020

(C) 2020, Skazkina V.V., Karavaev A.S., Borovkova E.I., Simonyan M.A., Prokhorov M.D., Ponomarenko V.I., Dubinkina E.S., Bezruchko B.P., Gridnev V.I., Kiselev A.R.

C 2020, Russian Open Medical Journal

\section{Abstract:}

The purpose of this work is to study the interaction between the autonomic regulatory loops of blood circulation from long time series. Methods - We simultaneously recorded four-hour signals of electrocardiogram and photoplethysmogram from the ear and finger of ten healthy adults. We determined the intervals of phase synchronization of the studied regulatory loops and analyzed the dependence of their length on the recording time. The deviations of the total percentage of phase synchronization (index $S$ ) from its mean value were estimated in moving non-overlapping windows.

Results - For studied signals we found no significant correlation between the length of synchronization epoch and the time of its beginning. A sharp increase in the deviation of the index $S$ from its mean was shown at the end of the experiment.

Conclusion - The increase in the deviation from the mean at the end of our records is most likely associated more with psychosomatic influences than with hormonal regulation or immobilization stress.

Keywords: autonomic control, heart rate variability, photoplethysmogram, phase synchronization, low-frequency oscillations.

Cite as Skazkina VV, Karavaev AS, Borovkova EI, Simonyan MA, Prokhorov MD, Ponomarenko VI, Dubinkina ES, Bezruchko BP, Gridnev VI, Kiselev AR. Uncovering interaction between the loops of autonomic regulation of blood circulation from long time series. Russian Open Medical Journal 2020 ; 9: e0403.

Correspondence to Anton R. Kiselev. Address: Research Institute of Cardiology, Saratov State Medical University, 112, Bolshaya Kazachya str., Saratov, 410012, Russia. Email: kiselev@cardio-it.ru.

\section{Introduction}

Some studies associate the development of stable oscillatory regimes in the heart rate with the interaction of several loops of autonomic regulation. Moreover, a change in the dynamics of such loops, compared, for example, with the evolution of pathological processes, led to a simplification of the dynamics of the heart rate and a decrease or disappearance of variability [1-5]. In the lowfrequency (LF) range $(0.04-0.15 \mathrm{~Hz})$, the loops of autonomic regulation of blood circulation provide normal control of heart rate, contractile force, vascular tone, and other processes of the cardiovascular system, which allow the system to flexibly adapt to the needs of organism. At the same time, the humoral regulation system also has an effect on the functioning of the circulatory system [6].

The interaction of loops of autonomic regulation of blood circulation in LF range is often described as the interaction of weakly coupled oscillators [7-12]. The peculiarity of such systems is that their interaction leads to a change in the signal phase, while the amplitude remains uncorrelated. The aim of this work was to study the dynamics of the interaction between the autonomic regulation loops over long periods using the methods of phase analysis. This work develops further the research described in Ref. [13].

\section{Material and Methods}

\section{Signal recording}

The experimental studies involved ten healthy volunteers aged 19 to 24 years. We carried out simultaneous registration of electrocardiogram (ECG) and photoplethysmogram (PPG) signals (signal of blood filling of the finger and ear, recorded by an optical sensor with active backlight in reflected light) of the vessels. The data were registered with a standard polyrecorder EEGA-21/26 "Encephalan-131-03" (Medikom MTD Ltd, Taganrog, Russia). The bandpass was $0.05-100 \mathrm{~Hz}$ and the sampling rate was $250 \mathrm{~Hz}$, digitized at 14 bits. ECG was recorded in the standard lead I according to Einthoven (differential signal from the wrists of two hands) and PPG was recorded from the distal (last, upper) phalanx of the ring finger and earlobe [14]. The PPG signal from a finger 
was recorded using a reflected light sensor in the IR range $(950 \mathrm{~nm})$ and the PPG from the ear was recorded using a reflected green light sensor $(560 \mathrm{~nm})[15,16]$.

The experimental data were recorded at rest in horizontal position. The length of each recording was 240 minutes. Figure 1 shows typical examples of experimental signals.

\section{Signal processing}

The study of heart rate variability from the ECG signals began with the extraction of the sequence of RR-intervals, i.e. a nonequidistant sequence of the time intervals between the R-peaks. Further, by the recommendation [17], we interpolated the obtained sequence using a cubic $\beta$-spline (Figure 1a).

The first step of the analysis included the estimation of epochs of phase synchronization between the LF-oscillations in RRintervals and PPG using a technique proposed in Ref. [18]. To calculate the phase of LF-oscillations in the experimental signal, we construct the analytic signal $\xi(t)$ for the signal $s(t)$ obtained as a result of bandpass filtration $(0.06-0.14 \mathrm{~Hz})$ of the experimental signal. The signal $\xi(t)$ is a complex function of time defined as follows:

$$
\xi(t)=s(t)+i \tilde{s}(t)=A(t) e^{i \varphi(t)},
$$

Where $A(t)$ and $\varphi(t)$ are, respectively, the amplitude and phase of the analytic signal and the function $\tilde{s}(t)$ is the Hilbert transform of $s(t)$

$$
\tilde{s}(t)=\frac{1}{\pi} \mathrm{P} \cdot \mathrm{V} \cdot \int_{-\infty}^{+\infty} \frac{s(\tau)}{t-\tau} d \tau_{x}
$$

where P.V. means that the integral is taken in the sense of the Cauchy principal value. Phase $\varphi(t)$ is defined from Eq. (1) as

$$
\varphi(t)=\arctan \frac{\tilde{s}(t)}{s(t)},
$$

Then we calculate the phase difference

$$
\Delta \varphi=\varphi_{1}-\varphi_{2},
$$

where $\varphi_{1}$ is the phase of LF-oscillations in RR-intervals and $\varphi_{2}$ is the phase of LF-oscillations of PPG.

The determination of the phase synchronization epochs was carried out using a linear approximation of the phase difference $\Delta \varphi$ in a moving window. The intervals with the phase difference value around some constant value $|\Delta \varphi|<$ const were diagnosed as phase synchronization intervals (4).
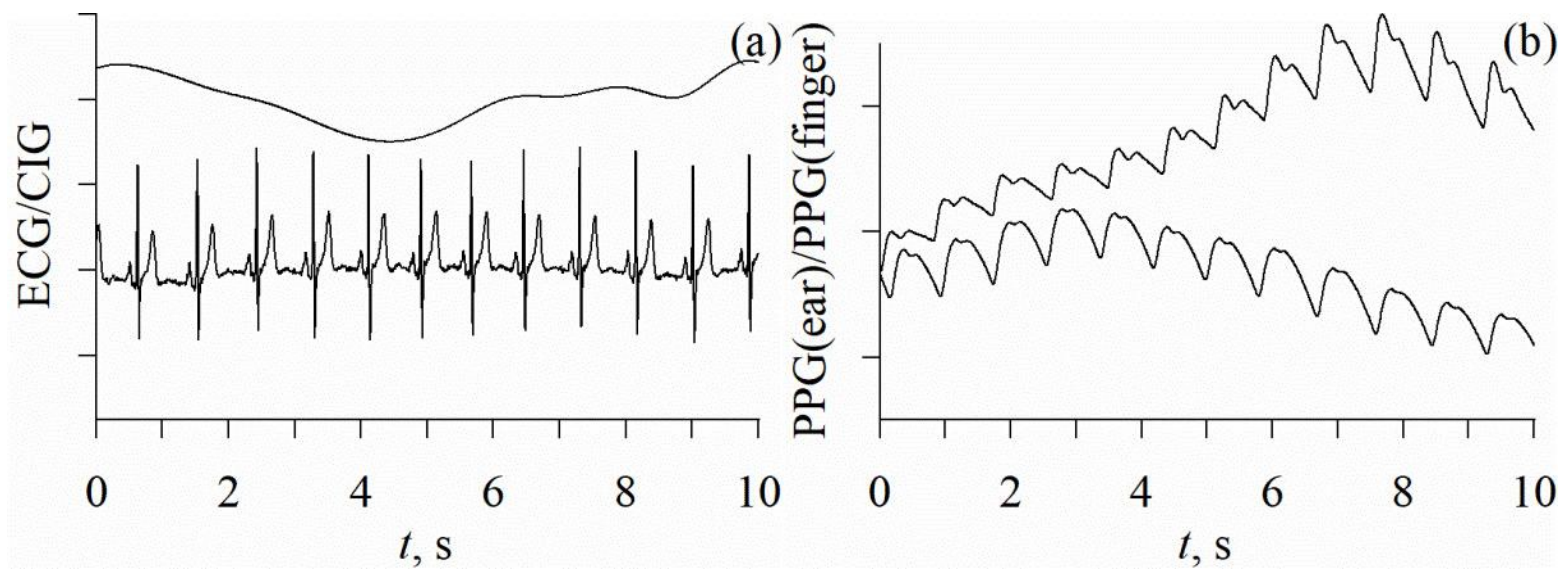

Figure 1. Typical examples of experimental data for ECG (bottom line) and interpolated sequence of RR-intervals (top line) (a) and PPG from ear (bottom line) and finger (top line) (b).
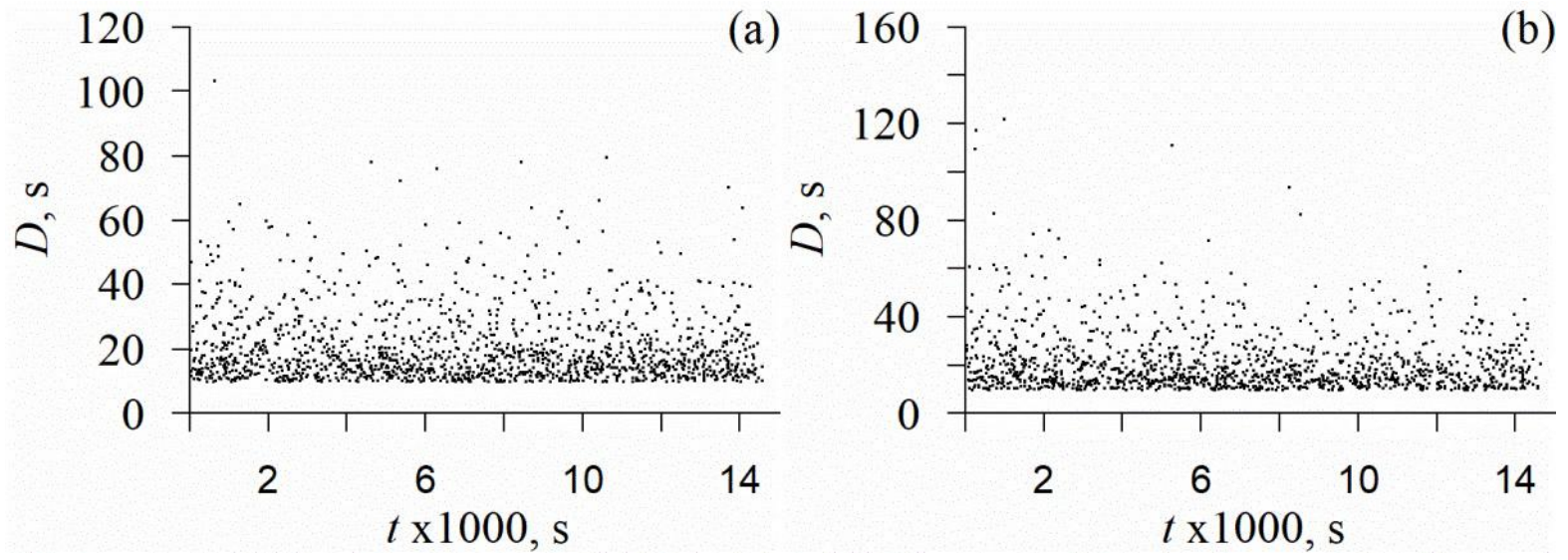

Figure 2. Dependences of the length $D$ of synchronization epochs on the time $t$ for the pairs of signals: RR-intervals and finger PPG (a), and RR-intervals and ear PPG (b). $t$ indicates the time of the beginning of synchronization epoch. 

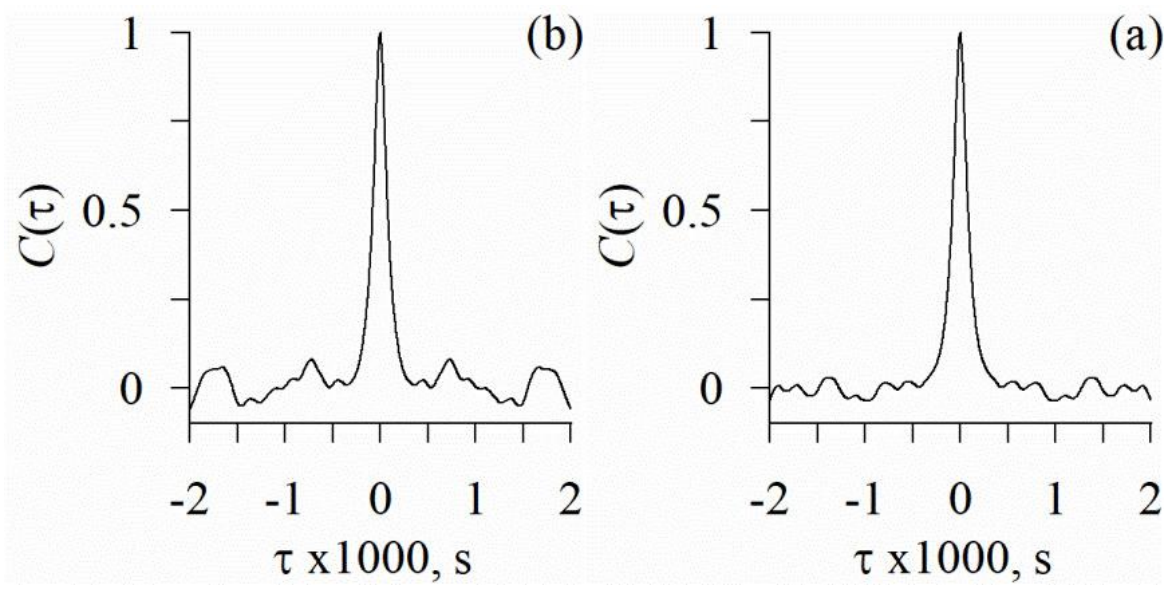

Figure 3. The autocorrelation function of $D(t)$, averaged over the entire experimental records of all subjects ( $n=10)$ for RR-intervals and finger PPG (a), and RR-intervals and ear PPG (b).
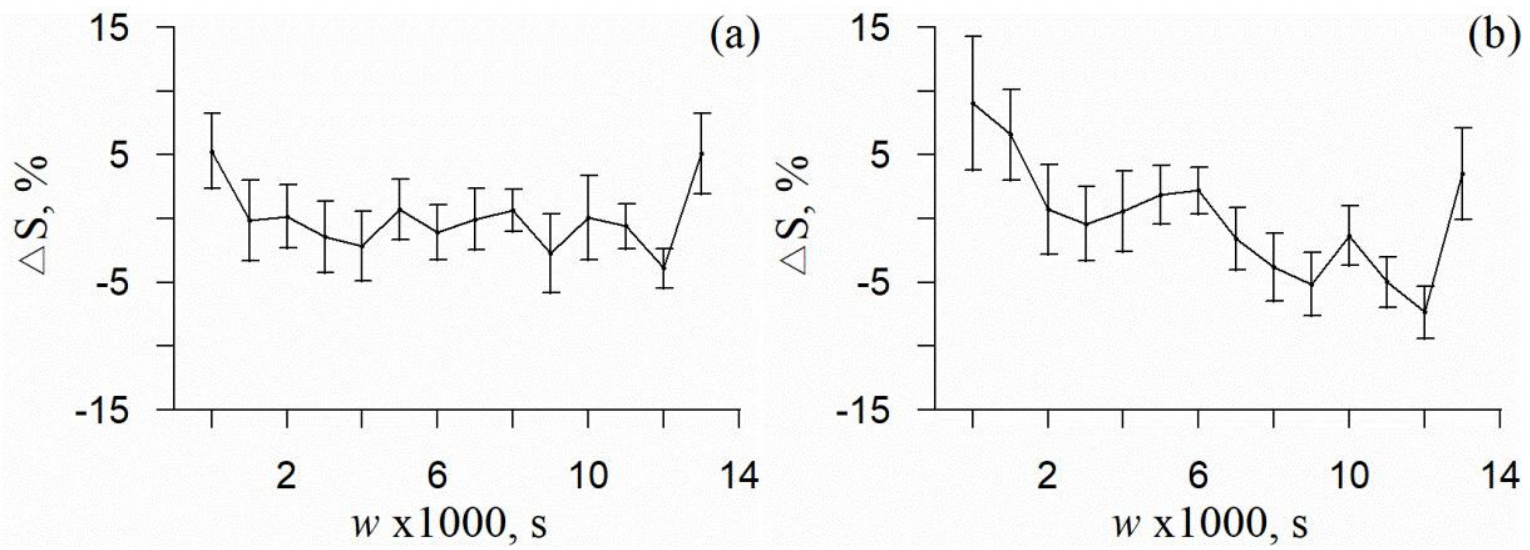

Figure 4. Averaged values of deviations from the mean $S$ value calculated in moving windows $w$. (a) RR-intervals and finger PPG; (b) RR-intervals and ear PPG. $w$ value corresponds to the middle of the interval used for the calculation of index $S$. The whiskers designate the standard deviation.

To quantitatively estimate the synchronization of the studied processes, we used the previously proposed total percentage of phase synchronization (index $S$ ). This index is the ratio of the sum of the lengths $d_{k}$ of the synchronization epochs to the total length $T$ of the recording:

$$
S=\frac{\sum_{k=1}^{N} d_{k}}{T} \times 100 \%
$$

where $k$ is the number of synchronization epoch and $N$ is the number of epochs. Index $S$ represents the percentage of time when the studied processes of autonomic regulation of circulation exhibited phase synchronization.

\section{Results}

In this work, we calculated the lengths $D$ of the synchronization epochs for the following pairs of signals: RRintervals and finger PPG (Figure 2a), and RR-intervals and ear PPG (Figure 2b). The points in Figure 2 show the lengths of epochs of synchronization for the entire ensemble of records.

It should also be noted that sufficiently long periods of phase synchronization are observed in healthy subjects. For example, Figure $2 \mathrm{~b}$ has a synchronization epoch between RR-intervals and ear PPG, which length is 123 seconds. However, we found no significant correlation between the length of the synchronization epoch and the time of its beginning.

We also calculated the autocorrelation function for all epochs of synchronization. These functions quickly fall to 0 , that is typical for a chaotic process (Figure 3).

Based on the results of Ref. [13], where two-hour signals were studied, we also estimated the total percentage of phase synchronization (index $S$ ).

Than we estimated the deviations $\Delta S$ from the mean $S$ value for each pair of signals: RR-intervals and finger PPG, and RRintervals and ear PPG. $\Delta S$ are calculated in moving nonoverlapping windows $w$ with a length of 1000 seconds. Figure 4 presents the dependences $\Delta S(w)$ averaged over the ensemble of records and the standard deviations.

\section{Discussion}

In Ref. [13], it was found out that the length of records for assessing the index $S$ should be at least 600 seconds. The deviation from the mean $S$ in moving windows (1000 s) was estimated from two-hour data. A small but significant decrease in the index $S$ from the mean value was observed in the middle of the second hour. After that, at the end of the record, $S$ returned to its value at the beginning of the experiment. To explain this process, we proposed 
a hypothesis about the effect of immobilization stress on the regulatory mechanisms of blood circulation due to prolonged immobility of the subject. It is also possible that the observed features are caused by impact of the humoral regulation system on the studied processes. In the present study, we use longer recordings to examine the correctness of these hypotheses.

Figure $4 \mathrm{~b}$ shows the greater change in the deviation of index $S$ from the mean value than Figure $4 a$. The difference between the Figure 4a and Figure $4 \mathrm{~b}$ may be associated with the peculiarities of local microcirculation, which we analyze by PPG from the ear. At the same time, PPG from the finger gives us mainly information about the mechanisms of the central nervous regulation of vascular tone [14-16]. It is worth paying attention to the sharp increase in the value of $\Delta S$ at the end of the record in both Figure 4a and Figure 4b. Earlier, we observed similar effect studying two-hour recordings [13] and assumed that it can be explained by the immobilization stress caused by a long stay in the immobile state and/or by slow humoral regulation. In this study, we increased the duration of the experiment to four hours, but the sharp increase in the value of $\Delta S$ was again observed at the end of the experiment. Probably, it may be associated with psychosomatic influences that could be triggered by reports of the remaining time of the experiment. We informed the subjects about the remaining time of recording every half hour of the experiment.

\section{Conclusion}

We studied the features of autonomic regulation of blood circulation using long-term four-hour simultaneous recordings of ECG and PPG signals. We estimated the lengths of epochs of phase synchronization between the LF oscillations in RR-intervals associated with the autonomic regulation of heart rate and both LF oscillations in finger PPG reflecting the tone of large vessels and LF oscillations in ear PPG reflecting the tone of small vessels and capillaries. For both pairs of signals, no significant correlation was found between the length of synchronization epoch and the time of its beginning.

We also tested the hypothesis about the increase in the average deviation of the total percentage of phase synchronization from the average value by the end of the two-hour experiment due to the influence of humoral regulation and/or the progress of immobilization stress [13]. In the present study, we observed an increase in the deviation of index $S$ from the mean value by the end of the four-hour experiment, which probably, may be associated with psychosomatic effects.

\section{Funding}

This study was funded by the Russian Science Foundation, Grant No. 19-12-00201.

\section{Ethical approval}

All procedures performed in the studies involving human participants were in accordance with the ethical standards of the institutional and national research committee and with the 1964 Helsinki declaration and its later amendments or comparable ethical standards. Design of this study was approved by the Ethics Committee of the Saratov State Medical University (Saratov, Russia) in 2019 and written informed consent was obtained from all subjects who participated in this study.

\section{Conflict of interest}

The authors declare that they have no conflict of interest.

\section{References}

1. Ishbulatov JM, Karavaev AS, Ponomarenko VI, Kiselev AR, Sergeev SA, Seleznev YP, et al. Phase synchronization of elements of autonomic control in mathematical model of cardiovascular system. Rus J Nonlin Dyn 2017; 13(3): 381-397. https://doi.org/10.20537/nd1703006.

2. Cavalcanti S, Belardinelli E. Modeling of cardiovascular variability using a differential delay equation. IEEE Trans Biomed Eng 1996; 43(10): 982-989. https://doi.org/10.1109/10.536899.

3. Ponomarenko VI, Prokhorov MD, Karavaev AS, Kiselev AR, Gridnev VI, Bezruchko BP. Synchronization of low-frequency oscillations in the cardiovascular system: Application to medical diagnostics and treatment. Eur Phys J Spec Top 2013; 222: 2687-2696. https://doi.org/10.1140/epjst/e2013-02048-1.

4. Karavaev AS, Kiselev AR, Gridnev VI, Borovkova El, Prokhorov MD, Posnenkova OM, et al. Phase and frequency locking of $0.1 \mathrm{~Hz}$ oscillations in heart rate and baroreflex control of blood pressure by breathing of linearly varying frequency as determined in healthy subjects. Hum Physiol 2013; 39: 416-425. https://doi.org/10.1134/S0362119713010040.

5. Kiselev AR, Khorev VS, Gridnev VI, Prokhorov MD, Karavaev AS, Posnenkova OM, et al. Interaction of $0.1 \mathrm{~Hz}$ oscillations in heart rate variability and distal blood flow variability. Hum Physiol 2012; 38: $303-$ 309. https://doi.org/10.1134/S0362119712020107.

6. Smolensky MH, Hermida RC, Portaluppi F. Circadian mechanisms of 24hour blood pressure regulation and patterning. Sleep Med Rev 2017; 33: 4-16. https://doi.org/10.1016/j.smrv.2016.02.003.

7. Pikovsky AS, Rosenblum MG, Osipov GV, Kurths J. Phase synchronization of chaotic oscillators by external driving. Physica $D$ : Nonlinear Phenomena 1997; 104(3-4): 219-238. https://doi.org/10.1016/S0167-2789(96)00301-6.

8. Kotani K, Struzik ZR, Takamasu K, Stanley HE, Yamamoto Y. Model for complex heart rate dynamics in health and diseases. Phys Rev E Stat Nonlin Soft Matter Phys 2005; 72(4 Pt 1): 041904. https://doi.org/10.1103/physreve.72.041904.

9. Kralemann B, Cimponeriu L, Rosenblum M, Pikovsky A, Mrowka R. Phase dynamics of coupled oscillators reconstructed from data. Phys Rev E Stat Nonlin Soft Matter Phys 2008(6 Pt 2); 77: 066205. https://dx.doi.org/10.1103/PhysRevE.77.066205.

10. Rosenblum MG, Pikovsky AS, Kurths J. Phase synchronization of chaotic oscillators. Phys Rev Lett 1996; 76(11): 1804-1807. https://doi.org/10.1103/PhysRevLett.76.1804.

11. Schäfer C, Rosenblum MG, Abel HH, Kurths J. Synchronization in the human cardiorespiratory system. Phys Rev E Stat Phys Plasmas Fluids Relat Interdiscip Topics 1999; 60(1): 857-870. https://doi.org/10.1103/PhysRevE.60.857.

12. Kiselev AR, Mironov SA, Karavaev AS, Kulminskiy DD, Skazkina VV, Borovkova El, et al. A comprehensive assessment of cardiovascular autonomic control using photoplethysmograms recorded from earlobe and fingers. Physiol Meas 2016; 37(4): 580-595. https://doi.org/10.20537/nd1801001.

13. Karavaev AS, Skazkina VV, Borovkova EI, Kiselev AR, Ponomarenko VI, Kulminskiy DD, et al. Statistical properties of the phase synchronization index of cardiovascular autonomic control contours. Russ Open Med J 2018; 7: e0403. https://doi.org/10.15275/rusomj.2018.0403.

14. Allen J. Photoplethysmography and its application in clinical physiological measurement. Physiol Meas 2007; 28(3): R1-R39. https://doi.org/10.1088/0967-3334/28/3/R01.

15. Bashkatov A, Genina E, Kochubey V, Tuchin V. Optical properties of human skin, subcutaneous and mucous tissues in the wavelength range from 400 to $2000 \mathrm{~nm}$. J Phys D: Appl Phys 2005; 38(15): 2543. https://doi.org/10.1088/0022-3727/38/15/004.

16. Sviridova N, Zhao T, Aihara K, Nakamura K, Nakano A Photoplethysmogram at green light: Where does chaos arise from? Chaos, Solitons and Fractals 2018; 116: 157-165. https://doi.org/10.1016/i.chaos.2018.09.016. 
17. Heart rate variability: standards of measurement, physiological interpretation and clinical use. Task Force of the European Society of Cardiology and the North American Society of Pacing and Electrophysiology. Circulation 1996; 93(5): 1043-1065. https://doi.org/10.1161/01.CIR.93.5.1043.

18. Karavaev AS, Prokhorov MD, Ponomarenko VI, Kiselev AR, Gridnev VI, Ruban El, et al. Synchronization of low-frequency oscillations in the human cardiovascular system. Chaos 2009; 19(3): 033112. https://doi.org/10.1063/1.3187794.

\section{Authors:}

Viktoriia V. Skazkina - PhD student, MSc, Researcher, Laboratory of Nonlinear Dynamics Modelling, Saratov Branch of the Institute of RadioEngineering and Electronics of Russian Academy of Sciences, Saratov, Russia; Researcher, Department of Dynamic Modeling and Biomedical Engineering, Saratov State University, Saratov, Russia. http://orcid.org/0000-0001-9380-8292.

Anatoly S. Karavaev - DSc, Professor, Department of Dynamic Modeling and Biomedical Engineering, Saratov State University, Saratov, Russia; Leading Researcher, Department of Innovative Cardiological Information Technology, Institute of Cardiological Research, Saratov State Medical University, Saratov, Russia; Senior Researcher, Laboratory of Nonlinear Dynamics Modelling, Saratov Branch of the Institute of RadioEngineering and Electronics of Russian Academy of Sciences, Saratov, Russia. http://orcid.org/0000-0003-4678-3648.

Ekaterina I. Borovkova - PhD, Researcher, Laboratory of Nonlinear Dynamics Modelling, Saratov Branch of the Institute of RadioEngineering and Electronics of Russian Academy of Sciences, Saratov, Russia; Researcher, Department of Innovative Cardiological Information Technology, Institute of Cardiological Research, Saratov State Medical University, Saratov, Russia; Assistant Professor, Department of Dynamic Modeling and Biomedical Engineering, Saratov State University, Saratov, Russia. http://orcid.org/0000-0002-9621-039X.

Simonyan, Margarita A. - MD, Researcher, Department of Atherocslerosis and Chronic Ischemic Heart Disease, Institute of Cardiological Research, Saratov State Medical University, Saratov, Russia; http://orcid.org/00000002-9866-3069.

Mikhail D. Prokhorov - DSc, Head of Laboratory of Nonlinear Dynamics Modelling, Saratov Branch of the Institute of RadioEngineering and Electronics of Russian Academy of Sciences, Saratov, Russia. http://orcid.org/0000-0003-4069-9410.

Vladimir I. Ponomarenko - DSc, Professor, Leading Researcher, Laboratory of Nonlinear Dynamics Modelling, Saratov Branch of the Institute of RadioEngineering and Electronics of Russian Academy of Sciences, Saratov, Russia. http://orcid.org/0000-0002-1579-6465.

Elizaveta S. Dubinkina - student of Department of Dynamic Modeling and Biomedical Engineering, Saratov State University, Saratov, Russia. https://orcid.org/0000-0002-4636-3937.

Boris P. Bezruchko - DSc, Professor, Leading Researcher, Laboratory of Nonlinear Dynamics Modelling, Saratov Branch of the Institute of RadioEngineering and Electronics of Russian Academy of Sciences, Saratov, Russia. http://orcid.org/0000-0002-6691-8653.

Vladimir I. Gridnev - MD, DSc, Professor of Department of Dynamic Modeling and Biomedical Engineering, Saratov State University, Saratov, Russia; Director of Institute of Cardiological Research, Saratov State Medical University, Saratov, Russia. http://orcid.org/0000-0001-68077934.

Anton R. Kiselev - MD, DSc, Professor of Department of Dynamic Modeling and Biomedical Engineering, Saratov State University, Saratov, Russia; Head of Department of New Cardiological Informational Technologies, Institute of Cardiological Research, Saratov State Medical University, Saratov, Russia. http://orcid.org/0000-0003-3967-3950. 